\title{
On Bayesian Scatterometer Wind Inversion ${ }^{1}$
}

\author{
Ad Stoffelen and Marcos Portabella \\ Royal Netherlands Meteorological Institute (KNMI) \\ Postbus 201, 3730 AE De Bilt, The Netherlands \\ Phone: +31 302206568 , Fax: +31 302210843 \\ E-mail: ad.stoffelen@,knmi.nl,portabel@,knmi.nl
}

\begin{abstract}
In a quest for a generic unbiased scatterometer wind inversion method, the different inversion procedures currently in use are revisited in this paper. A careful examination of both the errors in the wind and in the measurement domain, combined with the non-linear shape of the Geophysical Model Function, GMF, leads to a generic and novel Bayesian wind retrieval approach in the measurement domain. In this approach the shape of the GMF solution manifold in measurement space is more important than the specified noise. This shape is related to the system wind direction sensitivity, and when this sensitivity is uniform, realistic and precise wind direction distributions are retrieved, even when measurements lie far from the GMF manifold. A simplified measurement space transformation that produces such uniform sensitivity for the European Remote Sensing Satellite (ERS) scatterometer is presented and shown to have reduced wind direction bias compared to the more traditional

\footnotetext{
${ }^{1}$ IEEE Trans. Geosci. Rem. Sens., Vol. 44, No. 6, pp. 1523-1533, 2006. @ Institute of Electrical and Electronics Engineers.
} 
(measurement-noise normalized) inversion for ERS. Moreover, the simplified wind inversion reveals a similar performance to the current operational ERS wind inversion, but is potentially more generally applicable. The simplified method is then applied to SeaWinds but is ineffective. In this case the instrument geometry results in a low sensitivity to wind direction at a few specific directions. As a consequence, certain wind direction solutions remain favoured in the SeaWinds inversion.

\section{Introduction}

Spaceborne wind observations have proven important for a wide variety of applications, including nowcasting, short-range forecasting and mesoscale numerical weather prediction (NWP) data assimilation [1], [2], [3], [4]. In most applications, spatially correlated biases and ambiguity removal errors, often associated with wind field granularity [5], are rather detrimental since a wrong flow pattern may be inferred. On the other hand, pure random errors are generally less detrimental. In addition, the error analysis of wind data derived from spaceborne sensors requires special care due to non-linearities and ambiguities in the geophysical model functions (GMF) leading potentially to multiple solutions and biases in the wind retrieval. However, because of the different spatial and temporal sampling characteristics as well as the different error characteristics of the various spaceborne systems, their mutual wind performance comparison is not trivial. To assess the information content of spaceborne wind observations, the above uncertainties need to be quantified, which will allow us to better compare different wind sensors and design future and improved sensors. 
In scatterometer studies three noise sources are distinguished: i) due to the backscatter measurement process [6], often called instrument noise, ii) due to the combination of field of views with different azimuth angles, often called geophysical noise [5] [7], and iii) due to GMF errors. The first two classes of noise and perhaps a part of the third class can be well expressed in terms of the backscatter measurement and amount to typically several percent. Since the inversion combines several of these measurements, the resulting uncertainty in the retrieved wind solution should be minimal [5]. However, when scatterometer winds are validated, normal wind component error estimates are typically $1.5 \mathrm{~m} / \mathrm{s}$ [8], [9], i.e., $\sim 25 \%$ of the mean wind amplitude. This suggests that the GMF error is much larger than the backscatter errors [1]. This assessment is relevant for the inversion problem and is used in next section.

Oliphant and Long [10] derived performance measures for wind retrieval, using the so-called Cramer-Rao bound. This bound identifies the most accurate wind retrieval possible and can be used in simulation studies. However, they embark on limiting approximations in the derivation of the biased estimate. In this paper, we primarily focus on bias or systematic error as introduced by various retrieval objective functions for real scatterometer data.

The most general approach used for scatterometer inversion is the Bayesian approach. However, different sets of assumptions in the implementation of this approach are taken, leading to different algorithms [5], [11], [12]. In particular, one such approach leads to the socalled Maximum Likelihood Estimator (MLE) technique [11], which is used to process data from the National Aeronautics and Space Administration (NASA) scatterometer (NSCAT) [13] and the SeaWinds scatterometer [14]. Although MLE inversion provides satisfactory results in terms of general wind retrieval quality, a common problem of such technique is that certain wind directions are favoured in the inversion process, leading to some wind direction 
biases and artificial accumulations in the retrieved wind distribution [5], [15], [16], [17], [18]. In order to overcome this problem, one suggested approach is to compromise the GMF such that the resulting wind direction distribution looks more realistic [18].

In this work, we show that the problem of the artificial accumulations is primarily related to the sensitivity of the instrument to wind direction, which in turn is mainly driven by the geometry of the system, i.e., the set of incidence angles, azimuth angles, and polarisations associated with the measurements at any Wind Vector Cell, WVC. These characteristics should be accounted for in the wind inversion step. As such, this is an inversion problem rather than a forward (GMF) problem. For the ERS scatterometers [19] this has been recognised and a more sophisticated implementation of the Bayesian approach has been followed [5], [12].

The goal of this paper is to first exploit the full Bayesian approach in order to propose a generic scatterometer inversion procedure that accounts for the wind sensitivity (i.e., geometry) limitations of the measurement system. Subsequently, a simplified implementation is developed and tested for the ERS and SeaWinds scatterometers. 


\section{Inversion problem}

The scatterometer wind inversion problem has the objective of finding the most probable wind given a set of $\mathrm{N}$ backscatter measurements, $\sigma_{\mathrm{o}}^{\circ}$, i.e., maximise the conditional probability of the true vector wind, $\mathbf{v}$, given $\sigma_{\mathrm{o}}^{\circ}$

$P\left(\mathbf{v} \mid \boldsymbol{\sigma}_{o}^{o}\right)=\int_{\mathbf{v}_{\mathbf{s}}} P\left(\mathbf{v} \mid \mathbf{v}_{s}\right) \cdot P\left(\boldsymbol{\sigma}_{s}^{o} \mid \boldsymbol{\sigma}_{o}^{o}\right) d \mathbf{v}_{s}$

where the first and second terms in the integral respectively represent the uncertainty in the wind and measurement domain. For a given measurement geometry, the integral is over the 2dimensional manifold $\mathrm{S}$ in the $\mathrm{N}$-dimensional measurement domain, i.e., a surface, which is defined by all possible wind solutions, $\mathbf{v}_{\mathrm{s}}$, through the measurement space vector $\sigma_{\mathrm{s}}^{\circ}=$ $\operatorname{GMF}\left(\mathbf{v}_{\mathrm{s}}, \boldsymbol{\varphi}, \boldsymbol{\theta}, \boldsymbol{p}\right)$. The measurement geometry here comprises 3 vectors of length $\mathrm{N}$, which are the sets of incidence and relative azimuth angles, respectively $\theta$ and $\varphi$, and the radar polarisation $\boldsymbol{p}$. As stated in the introduction, the wind domain errors are much larger than the measurement space errors. In other words, the first probability term is a relatively broad function as compared to the second one, such that the wind errors, aligned along the manifold, are much larger than the measurement domain errors, which extend both along and across the manifold. The equation thus poses the challenge of integrating these large wind errors along the measurement space manifold S through the non-linear GMF. Pierson [11] does not use the concept of a manifold, but implicitly assumes the same uncertainty both along and across the surface S. Such assumption is inconsistent with the finding above. 
To provide a solution, Stoffelen and Anderson [1] split the problem by assuming that the term $P\left(\sigma_{s}^{\circ} \mid \sigma_{o}^{o}\right)$ can be approximated by an ambiguous set of Kronecker delta functions. Solving for $\sigma_{s}^{o}$ and then inverting the GMF will then provide unbiased wind estimates since the $P(\mathbf{v}$ $\mid \mathbf{v}_{\mathrm{s}}$ ) error distribution is by approximation normal and unbiased in the wind components [20], $[21]$.

Following [5] a Bayesian approach is then used for solving $\sigma_{s}^{\circ}$ from $P\left(\sigma_{s}^{\circ} \mid \sigma_{o}^{\circ}\right)$. From Bayes' theorem, we state that the probability of $\sigma_{\mathrm{s}}^{\circ}$ given a set of backscatter measurements is proportional to the probability of this set of backscatter measurements given $\sigma_{\mathrm{s}}^{\circ}$, multiplied by the prior $\sigma_{\text {s }}^{\circ}$ probability

$$
P\left(\boldsymbol{\sigma}_{s}^{o} \mid \boldsymbol{\sigma}_{o}^{o}\right)=P\left(\boldsymbol{\sigma}_{o}^{o} \mid \boldsymbol{\sigma}_{s}^{o}\right) \cdot P\left(\boldsymbol{\sigma}_{s}^{o}\right)
$$

Maximising this probability by varying $\sigma_{s}^{o}$ on the surface $\mathrm{S}$ for constant $P\left(\sigma_{s}^{o}\right)$ results in only a variable measurement error term $P\left(\sigma_{o}^{\circ} \mid \sigma_{S}^{\circ}\right)$, which can be expanded as the maximum likelihood estimator (MLE) commonly used for scatterometry [11], [22], [23]

$$
M L E=\frac{1}{N} \sum_{i=1}^{N}\left(\frac{\sigma_{o i}^{o}-\sigma_{s i}^{o}}{K p\left(\sigma_{X i}^{o}\right)}\right)^{2}
$$

where $N$ is the number of independent measurements, $i$ the measurement index, and $\sigma_{X i}^{\circ}$ is usually taken to be either $\sigma_{o i}^{o}$ or $\sigma_{s i}^{o}$. Here, $K p\left(\sigma_{X i}^{\circ}\right)$ is the expected Gaussian observation error (noise) and has the form of $K p_{i} \times \sigma_{X i}^{\circ}$, where $K p_{i}$ is a dimensionless value which is represented (for any given measurement $i$ ) in the following way [5] [10]

$$
K p=\sqrt{K p_{\text {geoph }}^{2}+K p_{\text {instr }}^{2}}
$$


where $K p_{\text {geoph }}$ is the so-called geophysical noise (see Fig. 1), i.e., caused by a spatial representativeness error due to the wind variability within the resolution cell and the nonuniform spatial averaging inherent in the radar measurement, and $K p_{\text {instr }}$ is the instrument noise, i.e., noise produced by the technical properties of the radar. Note that Eq. 4 should also include a GMF error term. Given many measurements, the GMF error is represented by the misfit between the measurements and the GMF manifold. Although the $\sigma_{s}^{\circ}$ misfit is generally small compared to $K p$, a misfit term could be added. [Note: strictly speaking, when assuming Gaussian errors and $\sigma_{X i}^{o}$ is $\sigma_{s i}^{o}$, a term $\ln \left(K p\left(\sigma_{s i}^{o}\right)\right)$ should be added to the right-hand side of Eq. 3 but this term is generally not significant and, as such, not used].

As noted, the MLE formulation (Eq. 3) is derived with the following set of assumptions:

- Measurement errors are uncorrelated,

- Their errors are Gaussian, and

- The a priori probability $P\left(\sigma_{s}^{\circ}\right)$ is constant.

The approximation of constant $P\left(\sigma_{s}^{\circ}\right)$ is problematic [5]. However, the use of Eq. 3 generally provides useful results in terms of wind speed retrieval accuracy. This is not the case for the retrieved wind direction, where biases are present (see [20] for ERS, and [24] and [10] for Seawinds). 


\section{$2.1 \quad$ Wind direction skill}

For a given scatterometer, its set of $\mathrm{N} \sigma_{i}^{\circ}$ measurements at a WVC number defines a GMF surface, $\mathrm{S}$, in a $\mathrm{N}$-dimensional $\sigma^{\circ}$ space for varying speed and direction. For the ERS triplets $\left(\sigma_{1}^{\circ}, \sigma_{2}^{\circ}, \sigma_{3}^{\circ}\right)$, this surface has the shape of a cone in the 3D space [5]. The cone is a doublefolded manifold, which implies dual ambiguity.

In [5], the effect of normalization in Eq. 3 is investigated for ERS through visualization of differently scaled 3D measurement spaces. This geometrical approach is motivated by the fact that minimising MLE is identical to finding the point on the GMF surface closest to the measurement triplet in the transformed axis system $\sigma_{i}^{\circ} / K p\left(\sigma_{X i}^{\circ}\right)$. Fig. 2a shows a cross section of the cone in which the backscatter measurements (triplets) are scaled by a fixed $K p$ value $^{1}$ (i.e., $K p_{\text {instr }}=0.05 ; K p_{\text {geoph }}=0$.) and Fig. $2 \mathrm{~b}$ shows the same cone slice without scaling and in a transformed space where the axes are $z_{i}=\left(\sigma_{i}^{o}\right)^{0.625}$. The slice is almost perpendicular to the cone, approximately at constant wind speed and mainly shows the variation in measurement points due to wind direction [5].

Stoffelen and Anderson [5] show that to be able to obtain both accurate wind directions and a realistic wind direction distribution after inversion, it is desirable that, given a uniform wind direction distribution, equal portions of the transformed $\sigma^{\circ}$ triplets are thrown onto equal wind direction intervals. This is the case if the GMF surface has constant curvature and is circular (Fig. 2b) rather than elliptic (Fig. 2a).

\footnotetext{
${ }^{1}$ Note that Portabella and Stoffelen [24] show that by using a more realistic Kp (i.e., they assumed a $0.05 K p_{\text {instr }}$ and, in addition, developed a simple geophysical noise model dependent on wind speed and incidence angle), the resulting cone sections are similar to Fig. 2 a.
} 
As such, when the cone is elliptic, certain wind directions are favoured in the retrieval process, leading to artificial accumulations in the retrieved wind direction distribution ${ }^{1}$ [5].

In order to illustrate the problem of the artificial accumulations, Fig. 3 shows a simple schematic representation of the cone section shapes ${ }^{2}$ discussed in Fig. 2. Fig. 3a represents a circular cone section (equivalent to Fig. 2b), which is subdivided in 8 identical sectors (equivalent to 8 identical wind direction intervals). Given a uniform wind direction distribution and the same noise both across and along the cone section, each sector will contain the same amount of points (triplets). Let's assume that the circle and the $y$ measurements including noise, compresses $50 \%$ in the y axis, resulting in an ellipse. Fig. $3 \mathrm{~b}$ shows the projection onto the same 8 sectors for the new shape, i.e., the ellipse. Since the closest distance of a measured point to the surface corresponds to the normal direction, the 8 sectors now divide the measurement space differently. It is obvious that these sectors do not contain the same amount of noisy measurement points anymore, i.e., sectors B, C, F, and G contain more points than sectors A, D, E, and H. As in [5], we confirm that a circular shape of the cone for a uniform wind direction distribution is equivalent to a constant $P\left(\sigma_{s}^{\circ}\right)$.

From the three example measurement points in Fig. 3, one may note that those points close to the surface have well-defined distance minima, while far away points are ill-determined and will thus be associated with relatively broad MLE local minima.

A second advantage of a circular ERS cone section is its low sensitivity to GMF errors as compared to the elliptic cone. For example, if the radius of the GMF cone fit is much too small compared to real data, still useful wind direction distributions could be obtained for a circular cone, but rather not for an elliptic cone.

\footnotetext{
${ }^{1}$ The same effect is seen for SeaWinds when using Kp normalization in Eq. 3 [22].

${ }^{2}$ In this simple example, we assume that the cone is a single folded manifold.
} 
Consequently, by transforming the measurement space and removing the $K p$ noise normalization from Eq. 3, the assumption of constant $P\left(\sigma_{s}^{\circ}\right)$ along the transformed circular cone becomes acceptable and the wind direction retrieval is improved. In other words, for ERS inversion, the shape of the GMF solution surface (i.e., cone shape) is more important than the information on the noise (i.e., $K p$ normalization).

Alternatively, in Eq. 2 one could model the $P\left(\sigma_{s}^{\circ}\right)$ modulation. However, $P\left(\sigma_{s}^{\circ}\right)$ depends on the wind and this sensitivity is known through the GMF by approximation. If uncertainties in the GMF and the GMF derivative were known precisely, then a sound equation could be derived. In practice, the accuracy of the computed GMF derivatives is limited. 


\section{Generic inversion}

As discussed in section 2, a space transformation, which makes true the assumption of constant $P\left(\sigma_{s}^{\circ}\right)$, i.e., $z$ space, is desirable for ERS. Since the aim of this work is to set a generic method for scatterometer wind retrieval, we should now try to determine the corresponding space transformation for NSCAT or SeaWinds. However, this is not straightforward. In contrast with ERS, where the transformation could be derived straightforwardly through visualisation of the 3-dimensional measurement space, NSCAT and Seawinds measurement systems are 4-dimensional.

We therefore propose to set a generic approach that numerically determines the best measurement space transformation. First, we analyse the sensitivity to wind direction of a scatterometer system and, in second instance, propose a simplified implementation, which is based on the scaling or weighting of the different scatterometer beams, in order to achieve constant $P\left(\sigma_{s}^{\circ}\right)$. Since an appropriate transformation is already known for ERS (i.e., $z$ space), we use the latter to validate the proposed method.

\subsection{Sensitivity analysis}

To look for constant $P\left(\sigma_{s}^{o}\right)$, it is of interest to relate its probability density $p\left(\sigma_{s}^{o}\right)$ to $p\left(\mathbf{v}_{\mathrm{s}}\right)$, i.e., $p\left(\sigma_{s}^{\circ}\right)=p\left(\mathbf{v}_{\mathrm{s}}\right)\left\|\partial \mathbf{v}_{\mathrm{s}} / \partial \sigma_{s}^{\circ}\right\|$ (e.g., [23], p.15), since the probability of a given infinitesimal set of 
points is the same in measurement space and in the wind domain. For small variations of $\mathbf{v}_{\mathrm{s}}$ we may assume $p\left(\mathbf{v}_{\mathrm{s}}\right)$ uniform or constant and the dependency of $\boldsymbol{\sigma}_{S}^{\circ}$ on wind speed to be quasi-linear. Then, it is the total wind direction sensitivity that mainly determines the modulation of $p\left(\sigma_{s}^{\circ}\right)$. Therefore, for different $\sigma^{\circ}$ beams, which depend on wind direction $\phi$, the constraint

$$
\left\|\frac{\partial \boldsymbol{\sigma}^{o}}{\partial \phi}\right\|=\sum_{i=1}^{N}\left(\frac{\partial \sigma_{i}^{o}}{\partial \phi}\right)^{2}=\text { constant }
$$

should be applied on total wind direction sensitivity, where $\mathrm{N}=3$ for $\mathrm{ERS}$, and $\mathrm{N}=4$ for NSCAT or SeaWinds ( $\mathrm{N}=2$ for SeaWinds outer swath). Note that wind direction $\phi$ is defined relative to the mean of the fore and aft azimuth viewing direction, but in the SeaWinds left swath 180 degrees is subtracted. When Eq. 5 is not true, we should seek a transformation of $\sigma^{\circ}$, i.e., $\sigma^{\circ}$, which satisfies the following:

$$
\left\|\frac{\partial \boldsymbol{\sigma}^{o /}}{\partial \phi}\right\| \approx \text { constant }
$$

in order to obtain a constant prior term in Eq. 2.

In the geometrical interpretation, as illustrated in Figs. 2 and 3, we demand that for subsequent wind direction changes, $\Delta \phi$, the distance traveled in $\mathrm{N}$-dimensional measurement space, $\left\|\Delta \sigma^{o}\right\|$, should be similar. Since, we do not pose any constraint on the direction of travel and the fact that the measurement space may have many dimensions, the constraint may still result in complex transformed manifold shapes, albeit more regular than the nontransformed manifold. 
For the ERS case, the left plots of Fig. 4 represent the total sensitivity (solid curves) of CMOD-5 GMF [25] as a function of relative wind direction for a few WVC numbers and wind speed values. The dotted, dashed, and dash-dotted curves represent the individual fore-, mid- and aft-beam sensitivities, respectively, which contribute to the total sensitivity (see Eq. 5). The straight line corresponds to the mean total sensitivity over all wind directions:

$$
\text { Mean }=\frac{1}{M} \sum_{j=1}^{M} \sum_{i=1}^{3}\left(\left.\frac{\partial \sigma_{i}^{o}}{\partial \phi}\right|_{\phi_{j}}\right)^{2}
$$

where $\mathrm{M}$ is the number of wind direction intervals, i.e., 360 in this case.

In line with the discussion in section 2.1, these plots also show that using $K p$ normalization in the MLE inversion (see Eq. 3) does not result in a circular cone (i.e., the solid curves are far from being straight lines). Note that wind direction maximum sensitivities, i.e., upwind (0), downwind (180), and crosswind (90 and 270), correspond to the top and bottom parts of the plots in Fig. 3. Thus, our goal is to flatten as much as possible the total sensitivity (solid) curves.

\section{$4 \quad$ Beam Weighting Method}

For ERS, the constraint of Eq. 6 is achieved by the z-transform. A simpler and potentially generic way to achieve more constant wind direction sensitivity is to scale (weight) the individual beam sensitivities such that the resulting total sensitivity is closest to its mean value. In other words, we minimize the distance between the solid curve (total sensitivity) and the straight line (mean value) by re-scaling the dotted, dashed and dash-dotted lines 
(individual beam sensitivities). As such, the cost function we want to minimize looks as follows:

$J=\frac{1}{M} \sum_{j=1}^{M}\left[\sum_{i=1}^{3}\left(\left.a_{i} \cdot \frac{\partial \sigma_{i}^{o}}{\partial \phi}\right|_{\phi_{j}}\right)^{2}-\text { Mean }\right]^{2}$

where $J$ is the cost function and $a_{i}$ the scaling or beam weighting coefficients. Note that since $a_{i}$ is not a function of direction $(\phi)$, the minimization is analogous to looking for a vector transformation $\sigma^{o /}=a \cdot \sigma^{o}$, which obeys Eq. 6 (see section 3.1).

Also note that we have chosen the squared distance (between total sensitivity and the mean) rather than the distance (see Eq. 8) for minimization since the results (not shown) reveal that the former is more effective than the latter in flattening the total sensitivity curve.

For every wind speed and WVC number, $J$ is minimized and a set of $a_{i}$ found. The minimization results in a clear single minimum (for every wind speed and WVC number), indicating that the method is robust and unambiguous. Note that in order to use the $a_{i}$ coefficients, an estimation of retrieved wind speed must be provided prior to inversion. As shown by [5], a good estimate can be derived from the averaged backscatter value of fore and aft beams. For more detailed information on the $a_{i}$ coefficients, see Portabella and Stoffelen [26].

The left plots from Fig. 4 show the sensitivity in $\sigma^{o}$ space (from here on referred to as " $K p$ norm", since it uses the derived $K p$ normalization in the MLE inversion) and the right plots show the sensitivity in $\sigma^{o /}$ space, i.e., after beam weighting (from here on referred to as "beam weighted norm"). By comparing either sides of Fig. 4, one can easily see that, in general, the beam weighting is effective in flattening the total sensitivity curve, i.e. making 
the cone more circular. In Figs. $4 \mathrm{a}$ and $4 \mathrm{c}$, fore and aft beams especially contribute to the peaks while the mid beam contributes to the troughs. As such, the beam weighting is most effective for these low and medium winds at the inner swath (WVC numbers 1-6) region (see top and mid plots). However, it is less effective for high winds and the outer swath (WVC numbers 13-19) region (see bottom plots). In this case, the individual beam sensitivities (see Fig. 4e) contribute in a similar way to the total sensitivity, and therefore scaling does not help much in flattening the total sensitivity curve.

Note further that the mean total sensitivity remains almost the same after beam weighting (compare straight lines at either sides of Fig. 4), denoting consistency in the beam weighting procedure.

\subsection{Validation}

In this validation, we mainly show results in terms of wind direction skill since the beam weighting procedure is expected to improve this component most. Moreover, the results (not shown) reveal no significant wind speed skill difference between the three inversion types tested here, i.e., $z$ space, $K p$ norm and beam weighted norm.

The schemes that approach a circular cone, i.e., $z$ space and beam weighted norm, are expected to perform better than the $K p$ norm scheme where the latter shows less circularity (more ellipticity), i.e., in the inner swath [26]. The results show somewhat better agreement with the European Centre for Medium-Range Weather Forecasts (ECMWF) model, in terms of wind direction retrieval, for $z$ space and beam weighted norm (root mean square or RMS 
difference of $25.99^{\circ}$ and $26.03^{\circ}$, respectively) than for $K p$ norm inversion (RMS difference of $\left.26.35^{\circ}\right)$, at the inner swath region.

As mentioned in section 2, the shape of the cone plays a crucial role in the wind direction retrieval. As the cone becomes more elliptic (less symmetric), certain directions are favoured in the retrieval process, causing in turn some artificial accumulations in the retrieved wind direction distributions. This systematic effect becomes more acute for points lying far away from the cone surface, i.e., for triplets with high MLE values (see Fig. 3).

Fig. 5 shows the wind direction distributions for triplets with normalised MLE larger than 0.7 (note that triplets with MLE larger than 9 are screened out) representing about $4 \%$ of the total amount of the ERS wind observations. Looking at the left plots, some systematic accumulations at certain wind directions are discernible in the ERS-retrieved distributions (dotted) with respect to the ECMWF distributions (solid). These accumulations are largest for $K p$ norm inversion (Fig. 5c) and smallest for $z$ space (Fig. 5a). Note the similar pattern in Figs. 5c and 4c. The beam weighting (Fig. 5e) reduces the problem with respect to the $K p$ norm inversion, although it is not as effective as the z-space transformation (Fig. 5a).

However, if we look at the inner swath distributions (right plots), the region in which the beam weighting is most optimal, it is clear that the beam weighted norm distribution (Fig. $5 \mathrm{f}$ ) is very similar to the $z$ space one (Fig. $5 b$ ), and much improved with respect to the $K p$ norm distribution (compare peaks at $0^{\circ}$ and $180^{\circ}$ in Figs. 5d and 5f). Small dissimilarities between the beam weighted beam and $z$ space results are apparent, for example in the $0-90$ degree interval. Note that although these triplets have high MLE values, they are still of reasonable quality (see reasonable agreement between ECMWF and ERS-retrieved distributions from top and bottom plots) and therefore very important to keep. 
Finally, we have also looked at the ERS inversion problem for low winds under $4 \mathrm{~m} / \mathrm{s}$ (not shown). The results show no significant differences between the three inversion types, and all show similar wind direction errors. The reason for this is that the backscatter noise for low winds is relatively high as compared to the size of the cone, leading to systematic errors in the wind direction assignment (accumulations in the wind direction distributions). However, for such substantial wind direction error, still a useful wind vector skill is achieved for these low speeds.

In summary, we conclude that although $z$ space is the most optimal transformation for ERS in terms of the wind direction retrieval, the proposed beam weighting method represents an improvement with respect to $K p$ norm and therefore an alternative to $z$ space. In contrast to the $z$ transform, the beam weighting method is more generic and may be applied to other scatterometer systems.

\section{$5 \quad$ SeaWinds geometry case}

The beam weighting method presented in section 4 is tested here for SeaWinds. Discussions on the wind direction retrieval limitations for the SeaWinds measurement geometry and ways to overcome such limitations are included in this section. 


\subsection{Beam weighting skill}

Similar to the ERS case, Eqs. 7 and 8 are used for SeaWinds beam weighting. Fig. 6 is similar to the left plots of Fig. 4 but for SeaWinds. It represents the total sensitivity (solid curves) of NSCAT-2 GMF ${ }^{1}$ [27] as a function of wind direction for a few WVC numbers and wind speed values, before beam weighting.

Comparing the left plots of Fig. 4 with Fig. 6, there are certain wind directions for SeaWinds (Fig. 6) with relatively very small total sensitivity compared to the total sensitivity maxima, whereas for ERS (Fig. 4) the sensitivity modulation is modest (i.e., smaller difference between minima and maxima, in comparison with SeaWinds plot). In general, this behaviour can be seen at any across swath position (WVC number) and any wind speed regime.

By looking at the individual beam sensitivities (non-solid curves) that contribute to the total sensitivity (solid curve) in Fig. 6, one can see that the main reason for the insensitive areas is the relative orientation of the set of SeaWinds beam views in a WVC. As such, the relative beam geometry in many WVCs (see, for example, Figs. 7b and 7c) leads to wind direction intervals where the sensitivity is minimum (and very small) for all four beams. The fact that the SeaWinds inner beams are H-pol plays a secondary role in the total sensitivity.

On the one hand, when the relative beam geometry is optimal, i.e., the minimum sensitivities of the different beam views are not aligned but rather out of phase, then the large upwinddownwind asymmetry of the H-pol beams results in wind direction regions with small (individual beam) sensitivity (e.g., in Fig. 6a, the inner beam sensitivity values plotted as dotted and dashed-dotted curves are very close to the $\mathrm{x}$ axis in the range $\left.\left[-90^{\circ}, 90^{\circ}\right]\right)$. This

\footnotetext{
${ }^{1}$ Note: the NSCAT-2 GMF provides the best results in the current operational KNMI inversion [22].
} 
subsequently results in very low total sensitivity regions (relative to the maxima) as well. In the case that the inner beams were V-pol, these regions would be more sensitive.

On the other hand, when the relative geometry is poor, i.e., the minimum sensitivities of all beams are approximately aligned (e.g., Fig. 6b), the behaviour of the H-pol beam sensitivities makes the very low sensitivity areas less pronounced, in comparison with a full V-pol system. That is, if in Fig. $6 \mathrm{~b}$ the dotted and dash-dotted lines would behave as the dashed and dashdot-dot-dotted lines, respectively (i.e., substituting H-pol by V-pol), the total sensitivity (solid line) modulation would be generally smaller than it is now.

In conclusion, for SeaWinds geometry and at nadir WVC numbers 29 to 48, the problem of the artificial wind direction accumulations is expected to be more accute for a full V-pol system than for a combined V-pol/H-pol system. However, the latter will contain more artificial accumulations than the former in the so-called sweet regions (WVC numbers 9 to 28 and 49 to 68). Moreover, because of the upwind/downwind asymmetry of the H-pol beams, the latter substantially reduce the ambiguity problem (better upwind/downwind discrimination) thus improving the wind direction retrieval. As such, a mixed V-pol/H-pol system on SeaWinds geometry generally improves wind retrieval as compared to a full V-pol system.

For SeaWinds, the beam weighting is ineffective (not shown). It turns out that the cost function (see Eq. 8) contains very broad minima. As such, the minimization procedure results in drastic changes in the beam weighting coefficients at ill-defined minima or arbitrary minima (not shown). Given the areas of (relatively) very small sensitivity as discussed above, this result may be anticipated. It is clear that there is no way to flatten the total sensitivity when such areas are present. Moreover, in the cases where the relative geometry is more optimal, up-weighting the H-pol sensitivity is pointless because of the already mentioned 
large upwind-downwind asymmetry (in Fig. 6a, a large weight in the dotted and dash-dotted lines will adequately increase the sensitivity around $45^{\circ}$ and $315^{\circ}$, but in turn will excessively increase the sensitivity around $130^{\circ}$ and $\left.230^{\circ}\right)^{1}$.

\subsection{Wind direction performance}

As discussed in section 5.1, the NSCAT-2 GMF total sensitivity as a function of wind direction is far from constant and the beam weighting does not effectively contribute to flattening the total sensitivity curve. Stoffelen and Anderson [5] showed that, for ERS, the peaks and troughs of the total sensitivity curve are correlated with "artificial" (nongeophysical) peaks and troughs in the retrieved wind direction distribution.

Fig. 7 shows the total sensitivity as a function of WVC number and the retrieved wind direction (with respect to the satellite flight direction) for a wind speed of $8 \mathrm{~m} / \mathrm{s}$. Note that the cross section at WVC number 41 in Fig. 7 is plotted as the solid curve in Fig. 6c. The sensitivity values are plotted in grey scale. The sensitivity peaks (white) and troughs (black) shift as we move across the swath (i.e., with WVC number), drawing a pattern that is directly related to the beam relative geometry change across the swath. A similar pattern is observed in the retrieved wind direction distributions shown in Fig. 8 (see also Ebuchi [18]). The relatively insensitive black areas in Fig. 7 correspond generally with either white or black narrow features in Fig.8, associated respectively with relative wind direction accumulation or suppression. This indeed confirms the correlation between the sensitivity and the artificial accumulation or suppression of retrieved directions for SeaWinds. For further reference, [28]

\footnotetext{
${ }^{1}$ Note that in this case (i.e., sweet swath regions), the beam weighting of a full V-pol system would be more effective since $\mathrm{V}$-pol beams have no upwind/downwind asymmetry.
} 
shows 2D sections through the 4D SeaWinds measurement space that may be related to both Figs. 7 and 8. Note that the NSCAT fixed antenna geometry is similar to the SeaWinds geometry at WVC number 13 (see Fig. 7) and, as such, similar wind direction retrieval problems are expected for NSCAT. This has also been shown by Ebuchi [16], [17].

In order to overcome the problem of the artificial accumulations, a suggested approach in scatterometry is to tune the GMF within the uncertainty of the collocation data set [18]. In other words, the GMF is compromised to compensate for the inherent SeaWinds geometry problem. However, the GMF fit (forward problem) and the wind retrieval (inversion problem) are two independent procedures. Therefore, by tuning the GMF sensitivities (i.e., forward problem) to avoid such artificial accumulations (i.e., inverse problem), one likely ends up with a modified GMF, which actually does not fit the measurements as well as the original GMF. Although this procedure can result in satisfactory wind direction distributions, there is a risk that the wind retrieval accuracy is compromised. Along these lines, a new Ku-band GMF was developed for SeaWinds on QuikSCAT, i.e., QSCAT-1. The latter was better than NSCAT-2 at removing the artificial wind direction accumulations. However, Portabella [24] shows that despite the artificial accumulations, the NSCAT-2 GMF produces better quality winds than the QSCAT-1 GMF.

In this study, we do not attempt to modify the GMF sensitivities to compensate for geometry but rather to scale (weight) the relative beam sensitivities in the inversion step such that no wind directions are favoured in the retrieval. The main difference in these approaches is that we still use the best GMF fit to the observations rather than a compromised GMF fit, but account for the GMF non-linearity in a wind inversion motivated by a Bayes derivation, thereby not a priori compromising the retrieval quality. For ERS, the beam weighting 
approach is effective. However, this is not the case for SeaWinds, since areas of relatively very small total sensitivity exist.

\section{Conclusions}

In scatterometry, elaborate procedures have been developed to determine how $\sigma^{\circ}$ depends on the wind, i.e., the forward problem. On the other hand, MLE inversion has been widely used to estimate the wind from a set of $\sigma^{\circ}$ measurements. A common problem of such MLE approach is that certain wind directions are favoured in the inversion process, leading to some artificial wind direction accumulations in the retrieved wind distribution, associated with biases and granular wind fields. In this paper, we first revisit the Bayesian scatterometer wind inversion problem and set up a generic framework to try and overcome such problem. Secondly, a first implementation is developed, called beam weighting method, and tested for the ERS and SeaWinds scatterometers.

Following a Bayesian approach, Stoffelen and Anderson [5] develop the prior probability term of the ERS wind retrieval problem and show that, in contrast with common NSCAT and SeaWinds retrieval algorithms, using no $K p$ noise normalization in the MLE inversion is most optimal. Without using $K p$ normalisation in the MLE inversion and by transforming the measurement space (i.e., using $z$ space), the cone shape in measurement space becomes circular and the wind retrieval improves. Moreover, in Stoffelen and Anderson [5] and in this paper it is shown that using a $K p$ norm in the MLE inversion is equivalent to transforming the cone surface in a rather elliptic shape, leading to artificial accumulations in the retrieved wind direction distributions. As such, for ERS inversion, the shape of the cone is more important 
than the backscatter noise properties. In this paper, we show that this shape is related to the total wind direction sensitivity, which, if uniform, avoids the artificial wind direction peaks.

The ERS case nicely illustrates the important role of sensitivity, rather than of noise, in avoiding inversion biases. In Figs $2 \mathrm{a}$ and $3 \mathrm{~b}$ the noise is essentially the same for all beams, while in Figs $2 \mathrm{~b}$ and $3 \mathrm{a}$ the width of the noise distribution differs by about a factor of two between vertical and horizontal. In fact, the modulation of wind direction sensitivity (Fig. 4) is quite comparable to this variation in noise. Sensitivity scaling clearly results in better wind direction distributions than noise scaling (Fig. 5).

An algorithm that seeks for the best measurement space transformation is presented. The method results in the weighting of the different beams by the integrated wind direction sensitivity. The correlation between the total sensitivity and the artificial (non-geophysical) peaks and troughs in the retrieved wind direction distribution is discussed. The beam weighting procedure is generally beneficial, though most effective, in terms of producing a more circular cone, at the inner swath (WVC numbers 1 to 6), in particular for low and medium winds, and less effective for high winds and outer regions (WVC numbers 13 to 19). The ERS beam weighting is validated against ECMWF winds, using as a reference the $z$ space and the $K p$ norm inversions. The main differences between the inversions lie in the wind direction domain. Although the highest wind direction skill corresponds to the $z$-space inversion, the beam weighting shows improvement with respect to $K p$ norm, especially in the inner swath where its skill is similar to the $z$ space skill. As such, the beam weighting method turns out to be a good alternative to the $z$-space inversion, and since it is generic in principle, we then test it for SeaWinds.

The beam weighting turns out to be ineffective for SeaWinds. This is mainly due to the relative geometry of the SeaWinds beams. Certain wind directions show low sensitivity for 
any given beam, thus making the weighting of the individual beam sensitivities ill-defined. Similar problems are expected for NSCAT because of its similarity to the SeaWinds instrument geometry at WVC number 13. Perhaps, the method may be more successful when the wind direction regions of very low sensitivity are excluded, but this was not tested here.

The wind retrieval objective function tested here is quadratic, i.e., the noise specification and prior probability term have no wind solution dependency, i.e., are non-adaptive. Adaptive methods not only require the use of the somewhat inaccurate wind-dependent GMF gradients at the solution point under consideration, but also the wind sensitivity of the other terms in the objective function. As such, they involve (uncertain) non-linear feedback loops and often result in biased or even unstable results. Another adaptive method consists in empirically deriving the normalised wind direction distribution of a retrieval scheme without a prior probability term (cf. Eq. 3) for an approximate class of wind speed and a given WVC. Subsequently, this distribution may be used as a prior term to effectively reduce (increase) wind direction probability at the initial peaks (gaps). Although potentially of interest, we did not exhaustively explore adaptive wind retrieval methods yet, since these include feedback loops and are more complex.

For SeaWinds, artificial accumulations in the retrieved wind direction distribution may still be avoided by improved wind retrieval. In this respect, Stiles et al. [29] and Portabella and Stoffelen [30] have developed different procedures that allow the broad MLE cost function minima, that are naturally associated with cases of low wind direction sensitivity, to be represented in the scatterometer ambiguity removal. These methods prevent the a priori selection of arbitrary and ill-defined MLE minima as the only ambiguous wind solutions to be presented to the ambiguity removal step. In particular, the Multiple Solution Scheme (MSS) [30] uses the full wind vector probability density function, $P\left(\boldsymbol{v} \mid \boldsymbol{\sigma}_{o}^{\circ}\right)$ conform Eq. (1) as 
computed from a set of backscatter data in a WVC, as input for the ambiguity removal step. Subsequently, the 2D Variational Ambiguity Removal (2D-VAR) ensures a meteorologically balanced wind field solution.

\section{Acknowledgments}

We greatly appreciate the valuable suggestions made by the reviewers that much improved this manuscript. This work is funded by the European Space Agency under the project contract number 18041/04/NL/AR, in collaboration with the Institute for Applied Remote Sensing (IFARS). The software used in this work has been developed through the European Meteorological Satellite Organization (EUMETSAT) Satellite Application Facilities (SAFs), involving colleagues at KNMI. The QuikSCAT data were obtained from the NASA Physical Oceanography Distributed Active Archive Center, at the Jet Propulsion Laboratory / California Institute of Technology, and the National Oceanic and Atmospheric Administration. 


\section{References}

[1] Stoffelen, A., and Anderson, D., "Ambiguity removal and assimilation of scatterometer data," Quart. J. R. Met. Soc., vol. 123, pp. 491-518, 1997.

[2] Stoffelen, A., and Van Beukering, P., "Implementation of improved ERS scatterometer data processing and its impact on HIRLAM short range weather forecasts," Report NRSP2/97-06, Beleidscomissie Remote Sensing, The Netherlands, 1997.

[3] Undén, P., Kelly, G., Le Meur, D., and Isaksen, L., “Observing system experiments with the 3D-Var assimilation system," Technical Memorandum No. 244, European Centre for Medium-Range Weather Forecasts (ECMWF), Reading, United Kingdom, 1997.

[4] Stoffelen, A., and Cats, G., The impact of SeaSat-A scatterometer data on high-resolution analyses and forecasts: The development of the QEII storm, Mon. Wea. Rev. vol. 119, 2794$2802,1991$.

[5] Stoffelen, A., and Anderson, D., "Scatterometer data interpretation: measurement space and inversion," J. Atmos. and Oceanic Technol., vol. 14(6), 1298-1313, 1997.

[6] Cavanié, A., "Evaluation of Kp on central and lateral antennas of NSCAT over arctic sea ice," Report presented at the $12^{\text {th }}$ ASCAT Science Advisory Group Meeting, Darmstadt, Germany, 16-17 October 1997.

[7] Figa, J., and Stoffelen, A., "On the assimilation of Ku-band scatterometer winds for weather analysis and forecasting", IEEE Trans. on Geoscience and Rem. Sens. (special issue on Emerging Scatterometer Applications), vol. 38 (4), pp. 1893-1902, 2000. 
[8] Stoffelen, A., "Towards the true near-surface wind speed: error modelling and calibration using triple collocation", J.Geoph. Res., vol. 103 (C4), pp. 7755-7766, 1998.

[9] Freilich, M. H., "Validation of vector magnitude datasets: effects of random component errors," J. Atmos. Oceanic Technol., vol. 14, pp. 695-703, 1997.

[10] Oliphant, T. E., and Long, D. G., “Accuracy of Scatterometer-Derived Winds using the Cramer-Rao Bound”, IEEE Trans. Geosci. Rem. Sens., vol. 37, no. 6, pp. 2642-2652, 1999.

[11] Pierson, W.J., "Probabilities and statistics for backscatter estimates obtained by a scatterometer," J. Geophys. Res., vol. 94, no. C7, pp. 9743-9759, 1989.

[12] Cornford, D., Csató, L., Evans, D. J., and Opper, M., "Bayesian analysis of the scatterometer wind retrieval inverse problems: some new approaches," J. R. Statist. Soc. B, vol. 66 (Part 3), pp. 1-17, 2004.

[13] Naderi, F. M., Freilich, M. H., and Long, D. G., "Spaceborne radar measurement of wind velocity over the ocean: an overview of the NSCAT scatterometer system," Proc. IEEE, vol. 79, pp. 850-866, 1991

[14] Spencer, M. W., Wu, C., and Long, D. G., "Tradeoffs in the design of a scapceborne scanning pencil beam scatterometer: application to SeaWinds," IEEE Trans. on Geoscience and Rem. Sens., vol. 35(1), pp. 115-126, 1997.

[15] Ebuchi, N., and Graber, H. C., "Directivity of wind vectors derived from the ERS-1/AMI scatterometer,” J. Geophys. Res., vol. 103(C4), pp. 7787-7798, 1998.

[16] Ebuchi, N., "Statistical distribution of wind speeds and directions globally observed by NSCAT,”J. Geophys. Res., vol. 104(C5), pp. 11393-11404, 1999. 
[17] Ebuchi, N., "Evaluation of NSCAT-2 Wind Vectors by Using Statistical Distributions of Wind Speeds and Directions," Journal of Oceanography, vol. 55, No. 5, pp. 575-584, 1999.

[18] Ebuchi, N., "Statistical distribution of wind directions observed by QSCAT," Proc. of fisrt QuikSCAT Cal/Val Workshop, Pasadena/Arcadia (USA), 1999.

[19] Attema, E. P. W., "The active microwave instrument on board the ERS-1 satellite," Proc. IEEE, vol. 79, pp. 791-799, 1991.

[20] Stoffelen, A., "Scatterometry," PhD thesis at the University of Utrecht, ISBN 90-3931708-9, 1998.

[21] Schyberg, H., and Breivik, L.-A., "Objective analysis combining observation errors in physical space and observation space," Research Report No.46, Norwegian Meteorological Institute, Oslo, Norway, 1997.

[22] Graham, R., Anderson, D., Hollingsworth, A., and Böttger, H., "Evaluation of ERS-1 wind extraction and ambiguity removal algorithms: meteorological and statistical evaluation," ECMWF report, ECMWF, Reading, United Kingdom, 1989.

[23] Chi, C., and Li, F., "A comparative study of several wind estimation algorithms for spaceborne scatterometers," IEEE Trans. Geoscience and Rem. Sens., vol. 26, no. 2, pp. 115$121,1988$.

[24] Portabella, M., "Wind field retrieval from satellite radar systems," PhD thesis at the University of Barcelona, ISBN 90-6464-499-3, available at http://www.knmi.nl/scatterometer, 2002.

[25] Hersbach, H., A. Stoffelen, and S. de Haan, "The improved C-band ocean geophysical model function CMOD-5”, submitted to J. Geophys. Res. - Oceans, \# 2005JC002931. 
[26] Portabella, M., and Stoffelen, A., "Study of an objective performance measure for spaceborne wind sensors," ESTEC contract, no. 18041/04/NL/AR, Netherlands, 2004.

[27] Wentz, F.J., and Smith, D.K., "A model function for the ocean normalized radar cross section at $14 \mathrm{GHz}$ derived from NSCAT observations," J. Geophys. Res., vol. 104(C5), pp. 11499-11514, 1999.

[28] Stoffelen, A., Voorrips, A., and de Vries, J., “Towards the Real_Time Use of QuikScat Winds”, BCRS project USP-2 report 00-26, ISBN 905411330 8, 2000.

[29] Stiles, B.W., Pollard, B.D., Dunbar, R.S., "Direction interval retrieval with thresholded nudging,” IEEE Trans. Geosci. Rem. Sens., vol. 40, no. 1, pp. 79-89, 2002.

[30] Portabella, M., and Stoffelen, A., "A probabilistic approach for SeaWinds data assimilation,” Quart. J. R. Met. Soc., vol. 130, no. 596, pp. 127-152, 2004. 


\section{List of Figures}

Fig. 1. Schematic illustration of scatterometer sampling. A target WVC (thick solid square) is sampled from two different perspectives, with a beam footprint indicated in solid and dashed, respectively. Both sample the sub-WVC wind variability in a different way and not uniform, leading to a so-called spatial representativeness error.

Fig. 2. Intersection of the cone with the plane $\sigma^{\mathrm{o}}{ }_{1}+{\sigma^{\mathrm{o}}}_{3}=2 \sigma_{\text {ref }}^{\mathrm{o}}$, with a thickness of $0.05 \sigma_{\text {ref }}^{\mathrm{a}}$ for WVC number 7, for values of $\sigma^{\mathrm{o}}$ ref, a reference value of the average fore and aft beam $\sigma^{\mathrm{o}}$, corresponding approximately to a speed of $8 \mathrm{~m} / \mathrm{s}$. In (a) $\sigma^{0}{ }_{1}, \sigma^{\mathrm{o}}{ }_{2}$, and ${\sigma^{\circ}}_{3}$ values are scaled by $K p$, equal to 0.05 , and in (b) the cross section is made in a transformed space where the axis are $z_{i}=\left(\sigma_{i}^{o}\right)^{0.625}$. The vertical axis represents the measured and scaled $\sigma^{\circ}{ }_{2}$, and the horizontal $\left(\sigma^{\mathrm{o}}{ }_{1}+\sigma^{\mathrm{o}}{ }_{3}\right) / \sqrt{ } 2$ [see also 5].

Fig. 3. Schematic representation of the cone section shapes discussed in Fig. 2. The top plot represents a circular cone section, which is subdivided in 8 identical angular sectors crossing perpendicular to the circle (equivalent to 8 identical wind direction intervals). Given a uniform wind direction distribution, each sector will contain the same amount of points (triplets). Three example measurement points are given by a plus, a circle and a star symbol. The bottom plot shows the normal projection of the measurement points onto the 8 angular intervals, obtained by compressing the $y$-axis in panel a) by $50 \%$. Since the compression is only in $y$, the vertical lines remain in the same $x$-axis position. The new sectors, now perpendicular to the ellipse, are not identical anymore and of the same three example points, 
two $(+, *)$ now reside in different wind direction sectors. As such, each sector will now contain a different number of points, notably sectors B, C, F, and G, contain more points than sectors A, D, E, and $\mathrm{H}$.

Fig. 4. ERS geometry with CMOD-5 total sensitivity (solid curves) as a function of wind direction for $\mathrm{Kp}$ norm (left plots) and beam weighted norm (right plots) for a few WVC or WVC numbers and wind speed values: WVC number 3 and $4 \mathrm{~m} / \mathrm{s}$ (top plots), WVC number 5 and $8 \mathrm{~m} / \mathrm{s}$ (mid plots), and WVC number 13 and $15 \mathrm{~m} / \mathrm{s}$ (bottom plots). The dotted, dashed, and dash-dotted curves represent the individual fore-, mid- and aft-beam sensitivities, respectively. The straight line corresponds to the mean total sensitivity over all wind directions.

Fig. 5. Wind direction (with respect to the satellite flight direction) distribution of the ERSretrieved solution closest to ECMWF (dotted) for $z$ space (top), $K p$ norm (mid), and beam weighted norm (bottom) and for the inner swath (right) and the entire swath (left) over one month (January 1993). The solid line corresponds to the ECMWF wind direction distribution. The distributions only contain the $4 \%$ of triplets with MLE larger than 0.7 . The direction binning is $5^{\circ}$.

Fig. 6. SeaWinds geometry with NSCAT-2 total sensitivity (solid curves) as a function of wind direction, before beam weighting, for a few WVC numbers and wind speed values: WVC number 11 and $4 \mathrm{~m} / \mathrm{s}$ (top plot), WVC number 41 and $8 \mathrm{~m} / \mathrm{s}$ (mid plot), and WVC number 61 and $15 \mathrm{~m} / \mathrm{s}$ (bottom plot). The dotted, dashed, dash-dotted, and dash-dot-dotdotted curves represent the individual fore-inner, fore-outer, aft-inner, and aft-outer beam sensitivities, respectively. The straight line corresponds to the mean total sensitivity over all wind directions. 
Fig. 7. SeaWinds geometry with NSCAT-2 total sensitivity as a function of WVC number and wind direction for a wind speed of $8 \mathrm{~m} / \mathrm{s}$. The sensitivity values are plotted in grey scale, ranging from black (troughs) to white (peaks).

Fig. 8. Retrieved relative wind direction distribution as a function of WVC number and European Centre for Medium-range Weather Forecasts (ECMWF) 12-hourly predicted wind direction. The retrieved SeaWinds distribution is divided by the ECMWF distribution to compute the relative distribution. Bins below values of 0.6 (minimum at 0.2 ) are black, whereas white occurs above 2.0 (maximum at 5.6) with a logarithmic grey scale in between. Only WVCs 9-68 are processed. 
Illustrations

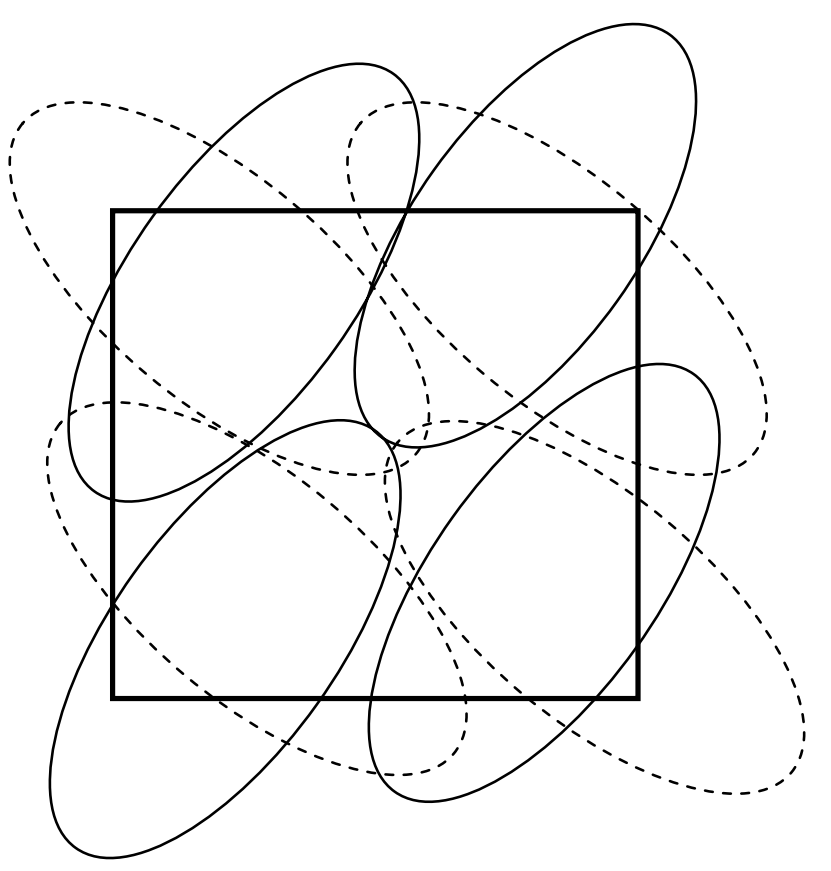

Fig. 1. 
a)

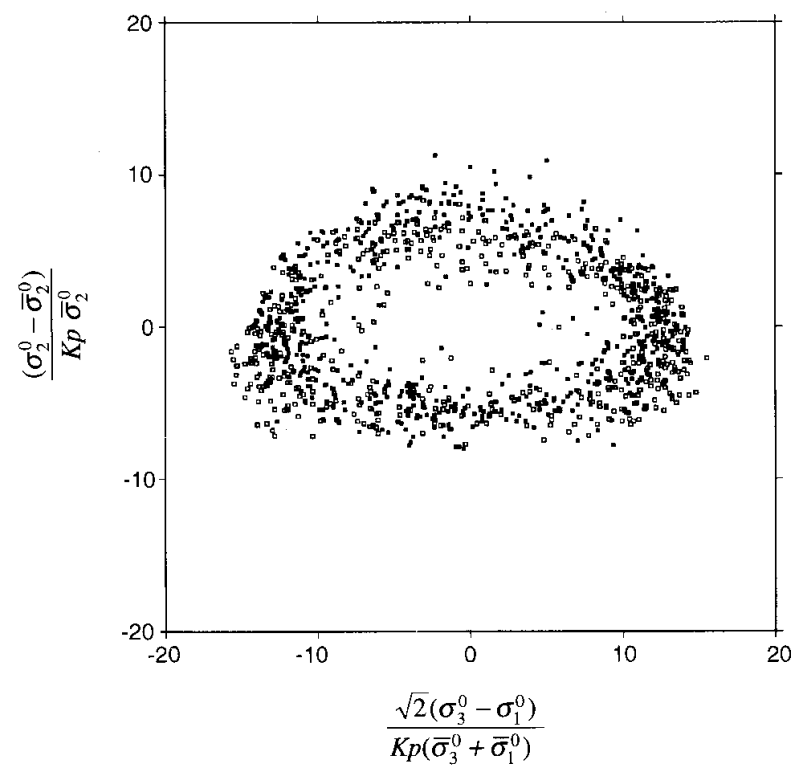

b)

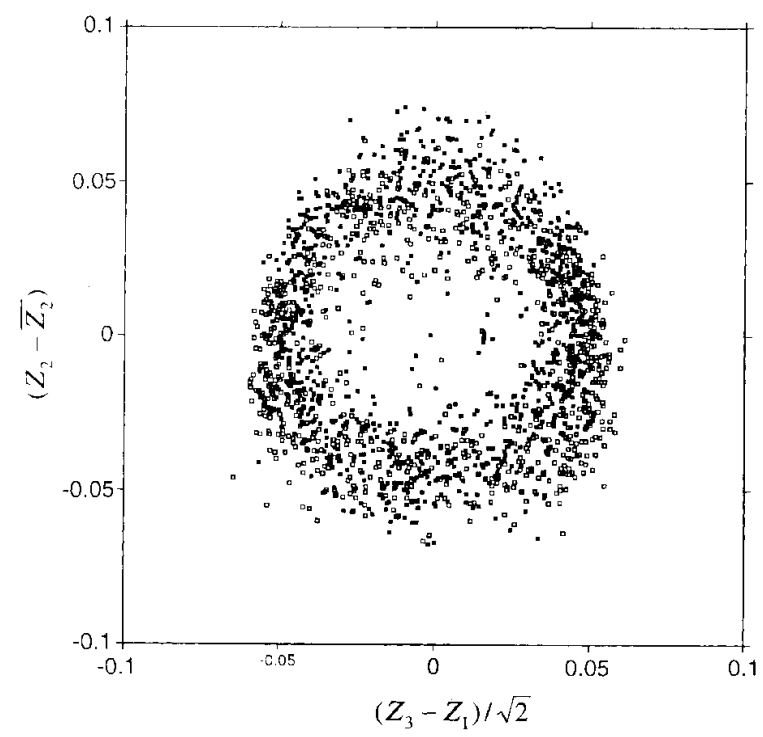

Fig. 2. 
a)

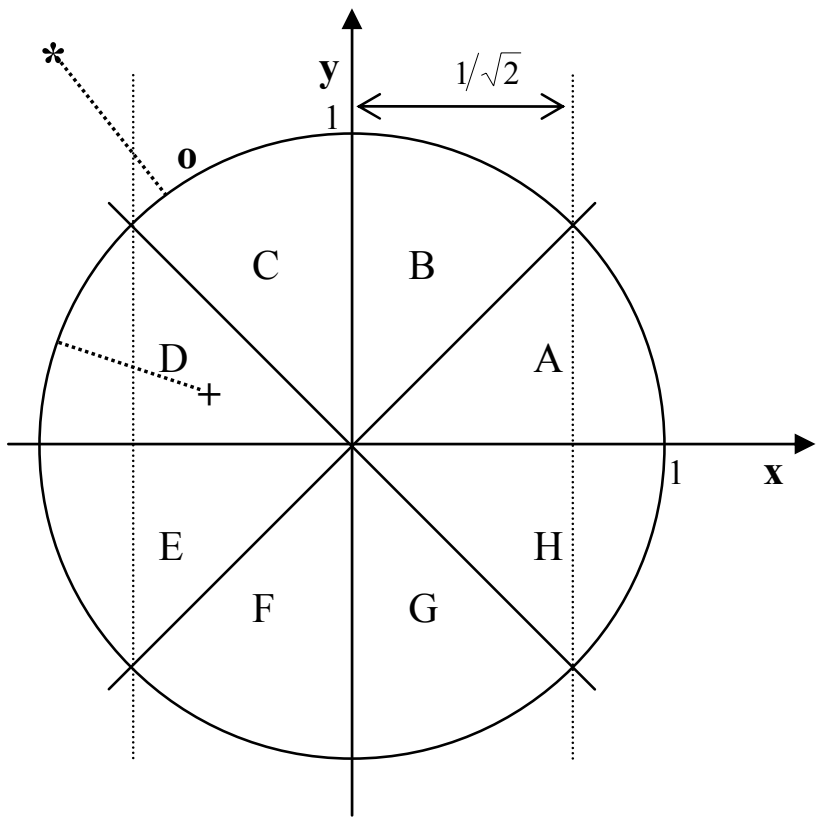

b)

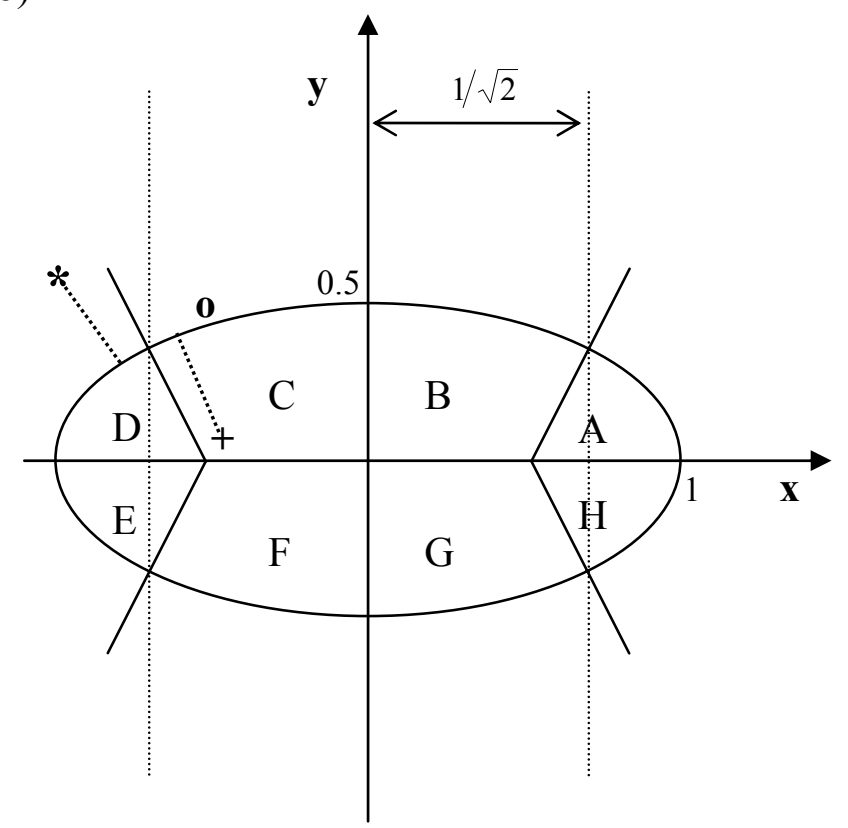

Fig. 3. 
a)

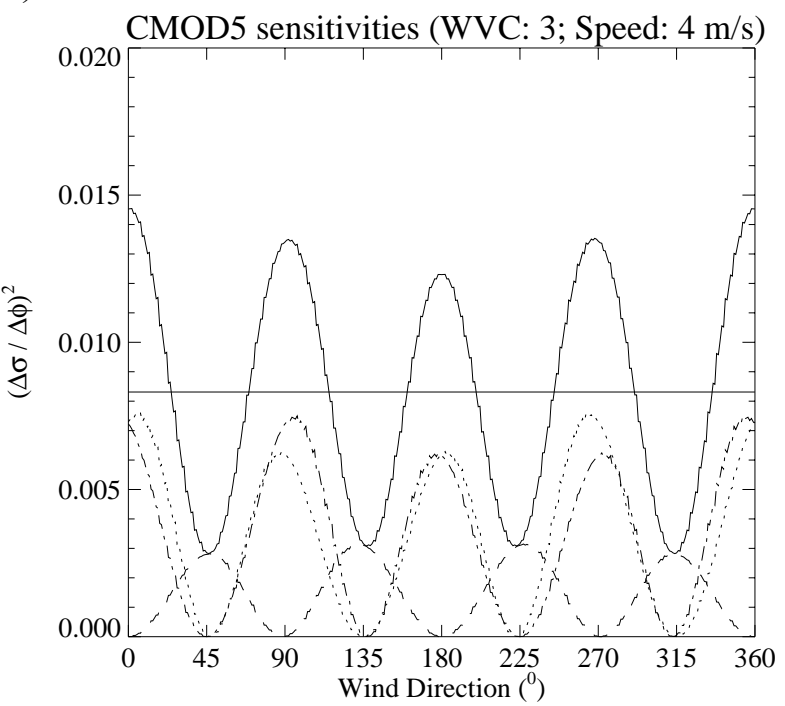

c)

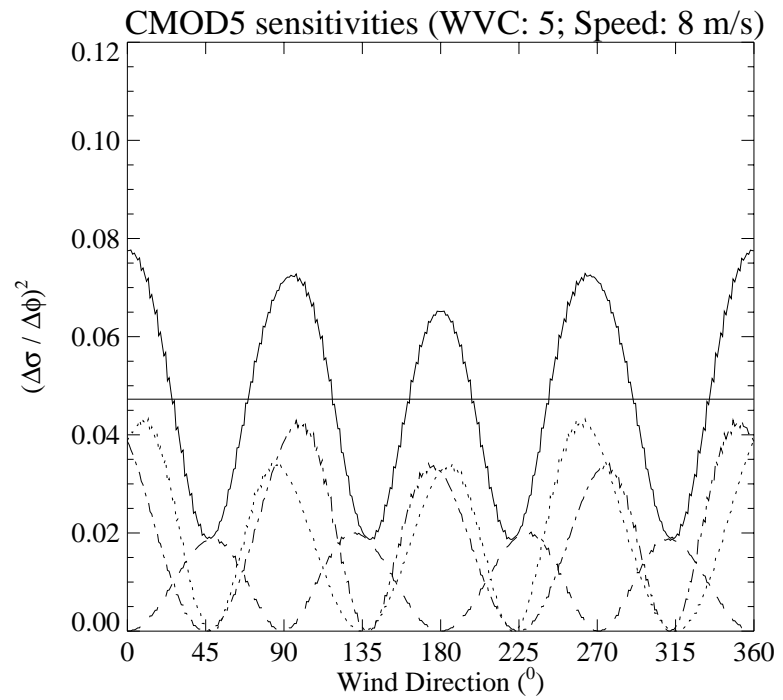

e)

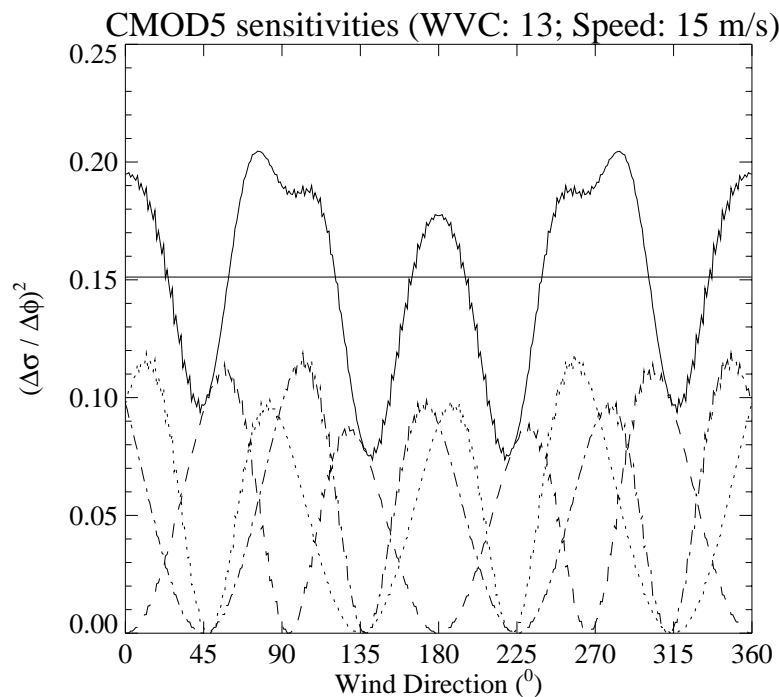

b)

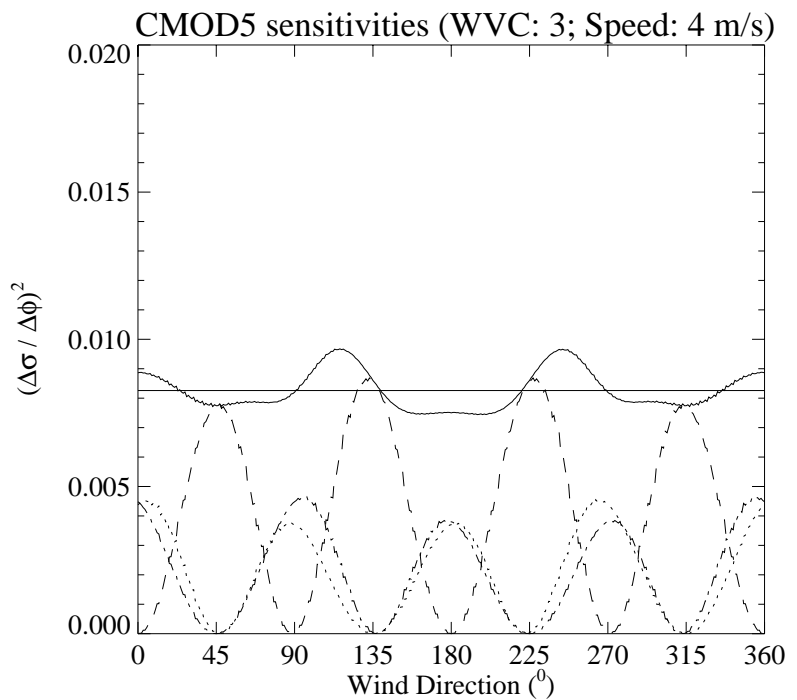

d)

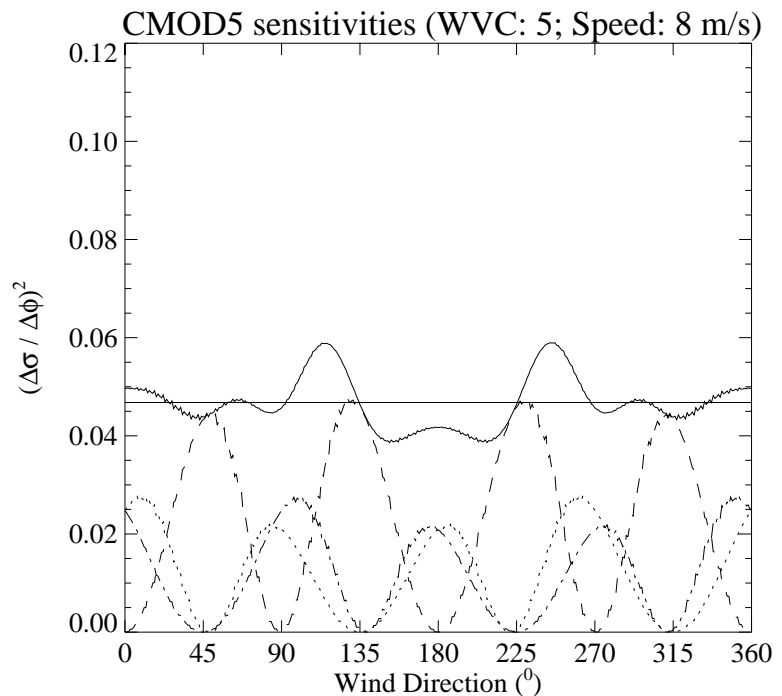

f)

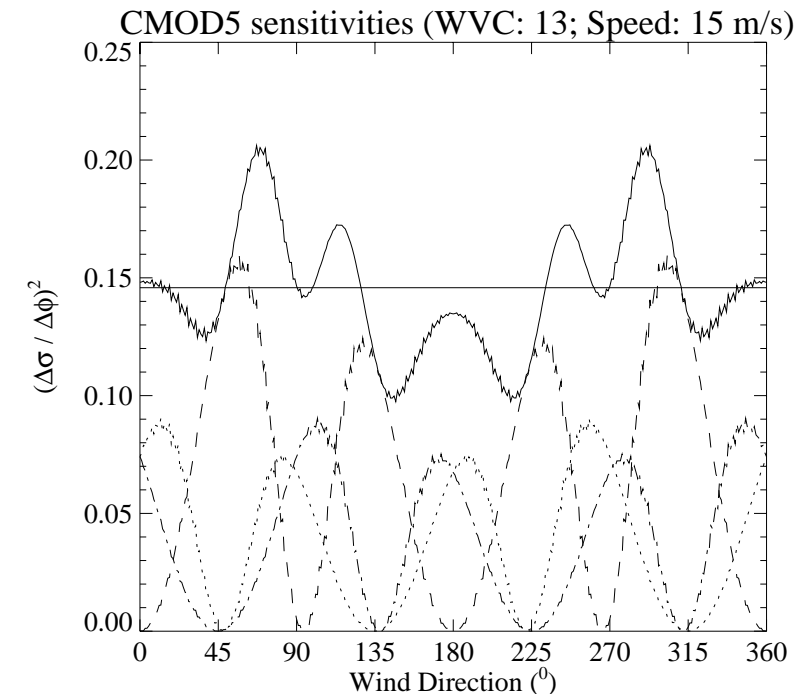

Fig. 4. 
a)

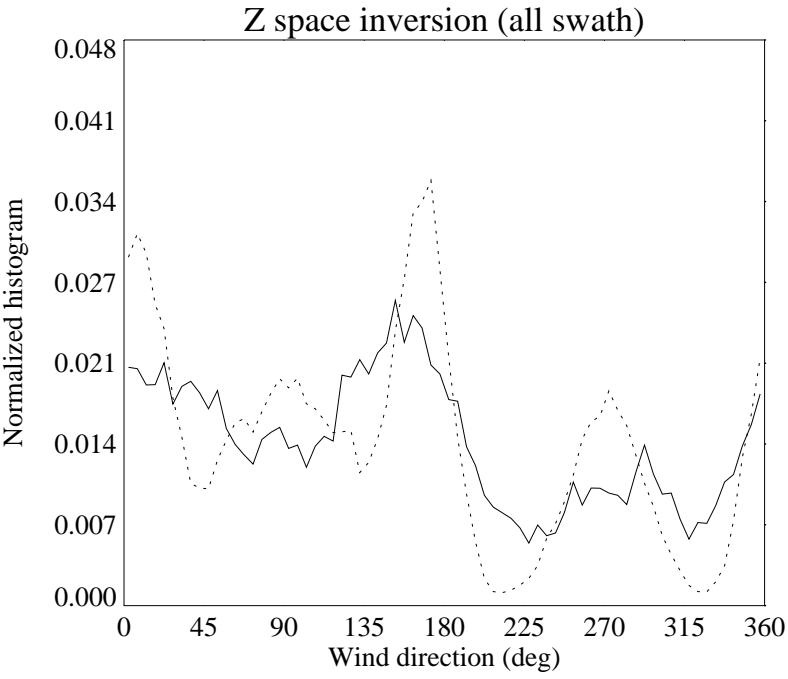

c)

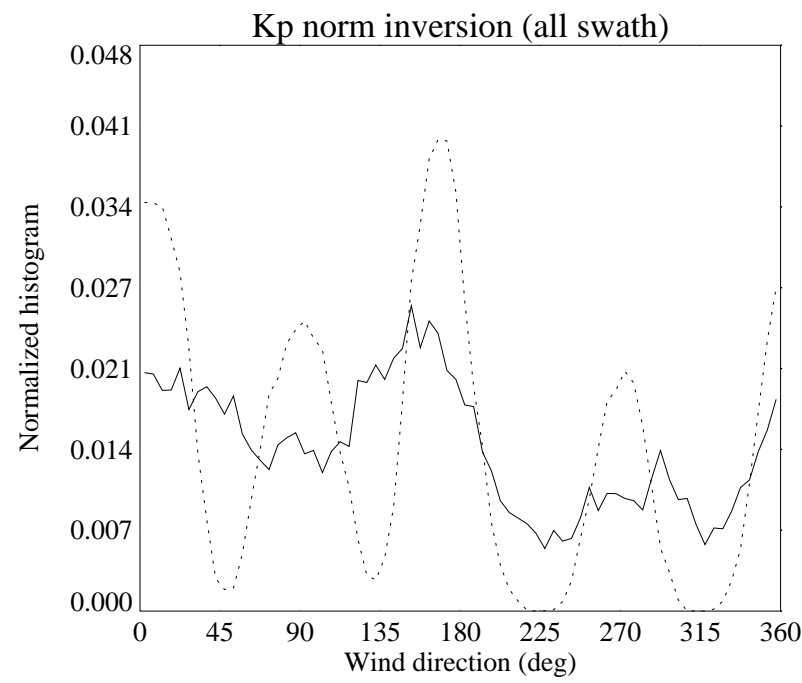

e)

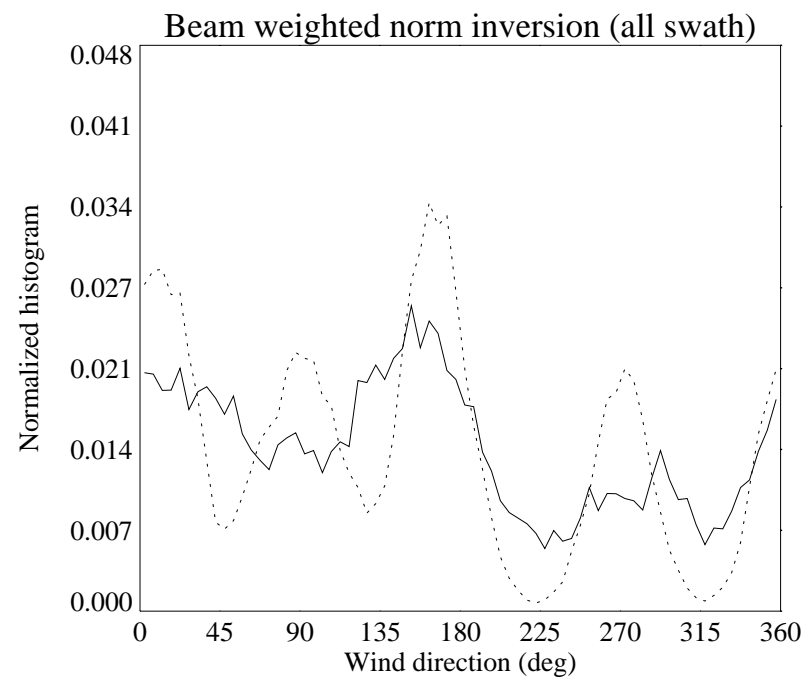

b)

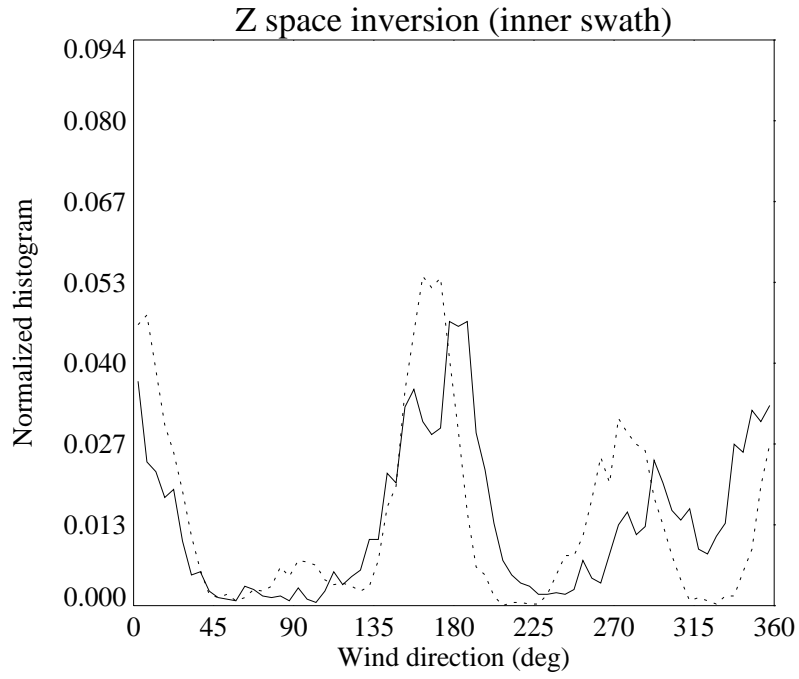

d)

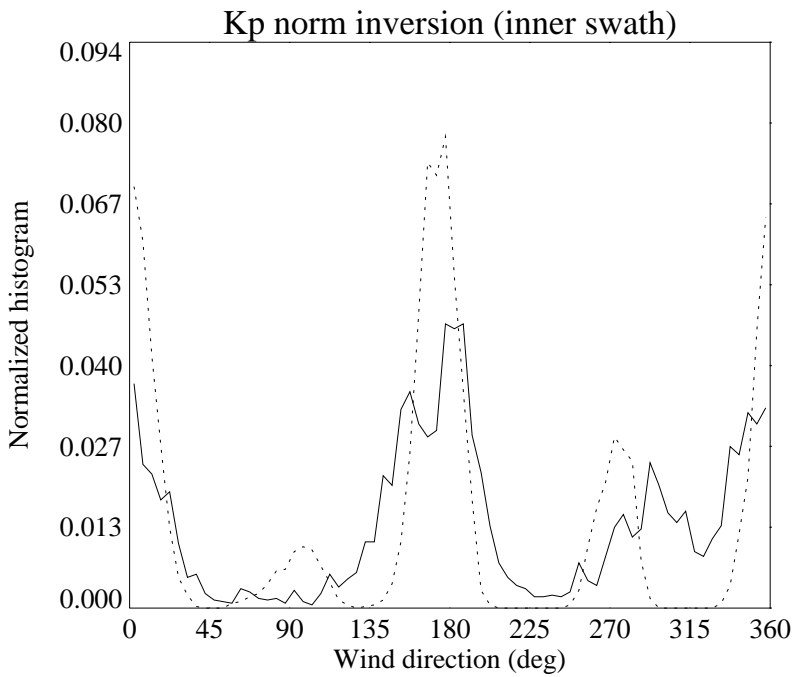

f)

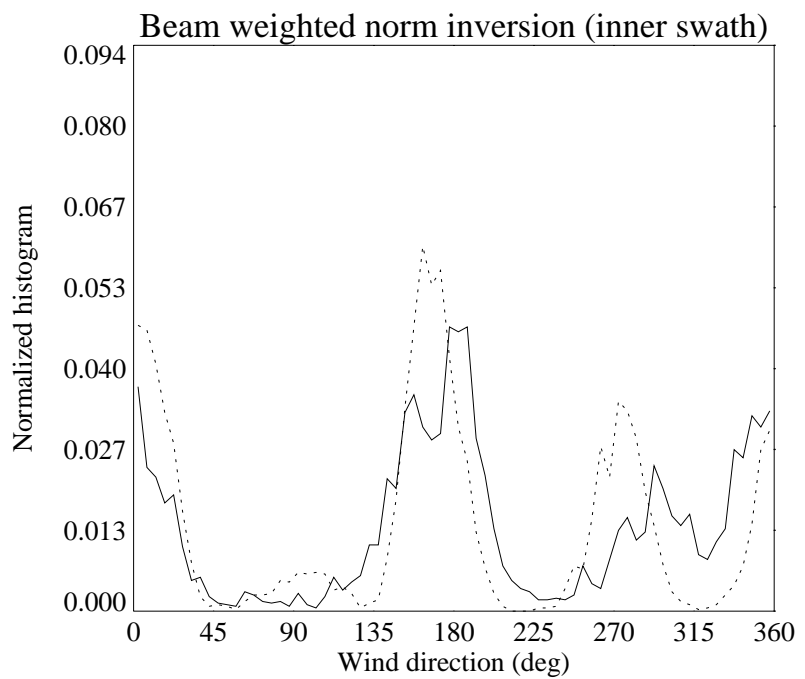

Fig. 5. 
a)

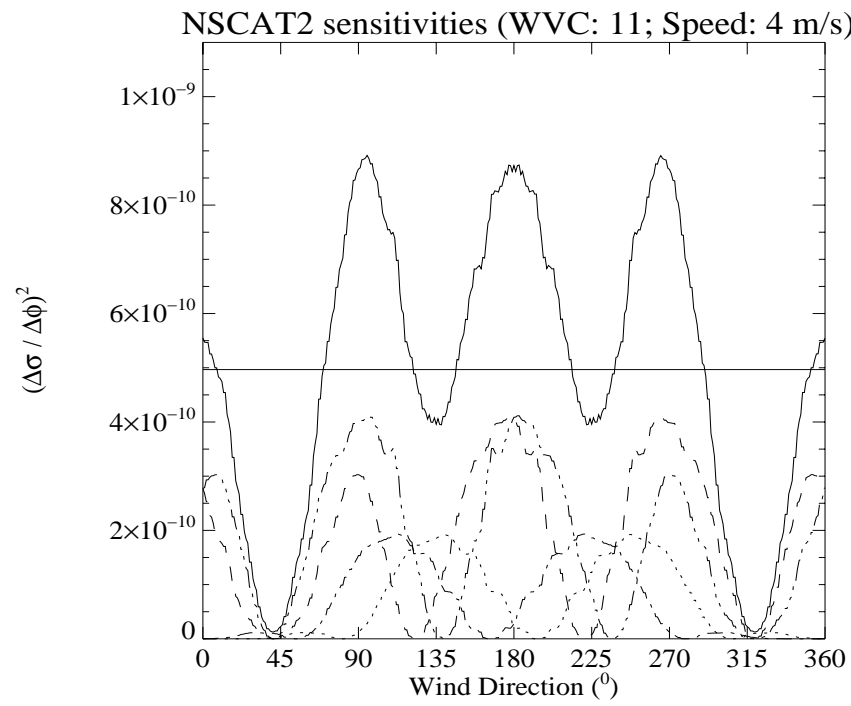

b)

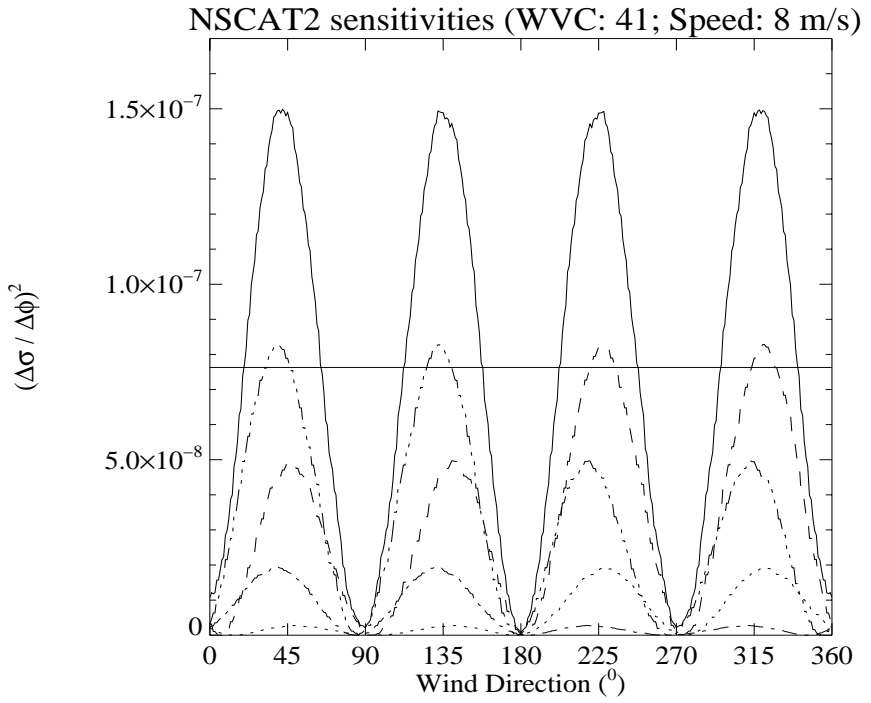

c)

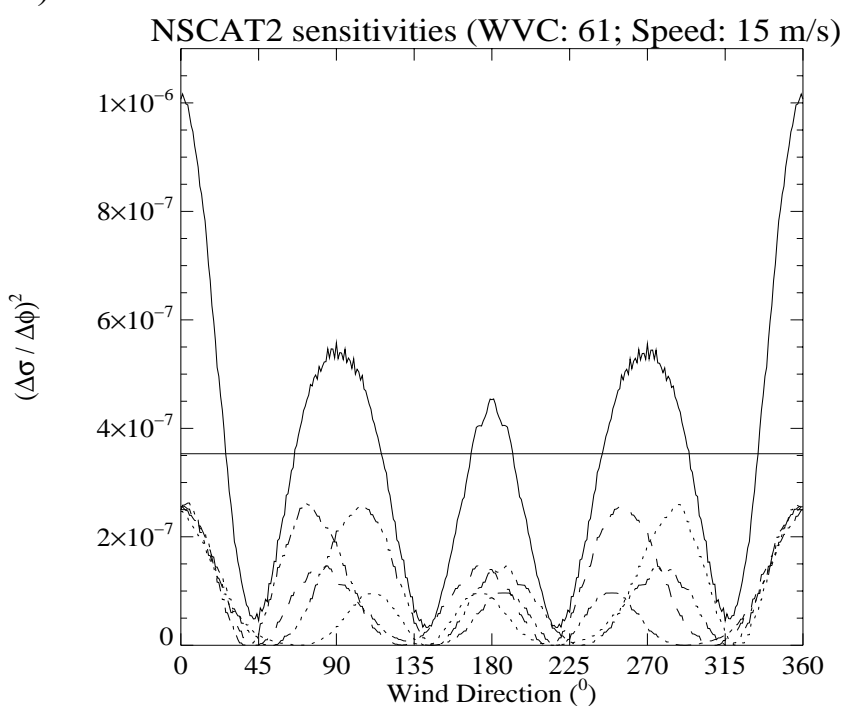

Fig. 6. 


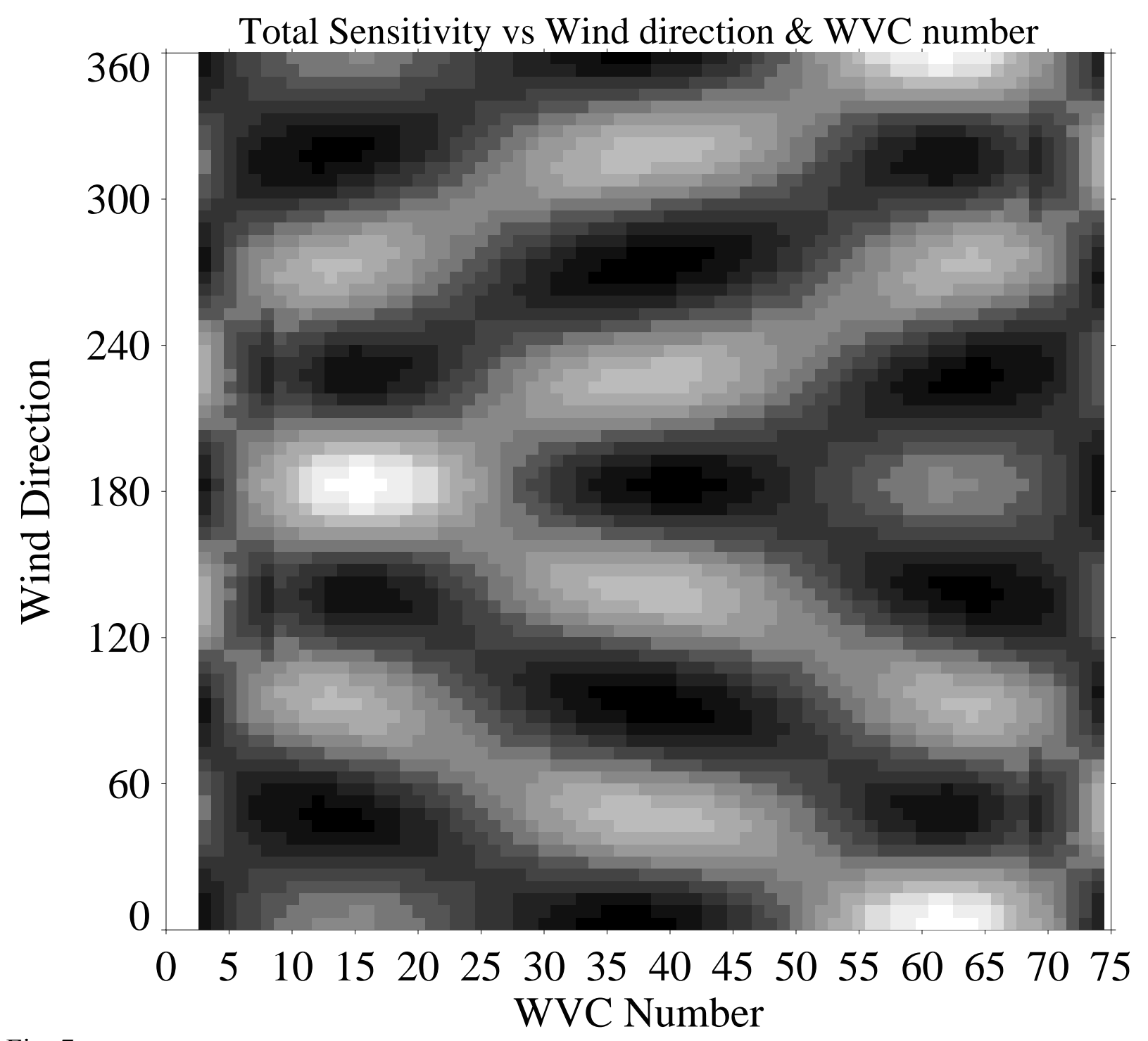

Fig. 7. 


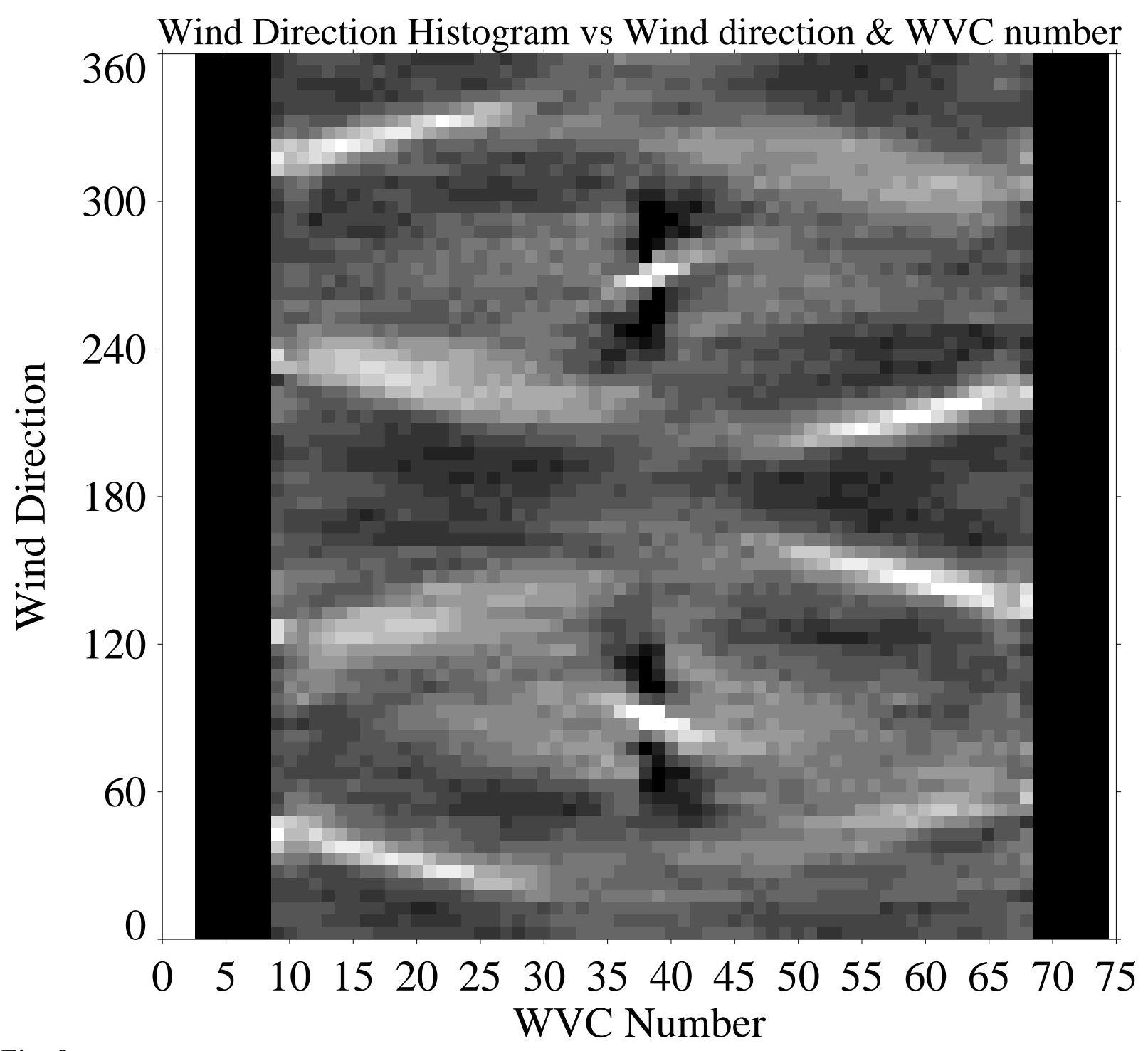

Fig. 8. 